\title{
Back to „First down“
}

\begin{abstract}
FALLBERICHT: AMERICAN-FOOTBALL-SPIELER MIT RÜCKENSCHMERZEN Fehlende Richtlinien für den RTP bei Low Back Pain, auf starren Zeitvorgaben basierenden Protokollen und fehlende Evidenz für die Assessments erschweren es Therapeuten, den passenden Behandlungsplan zu entwickeln. Warum also in der Therapie nicht einfach an Symptomen und Mechanik orientieren? Genau das macht ein McKenzie-Therapeut, zu dem ein junger Footballspieler mit LBP kommt. Die Parameter des Therapeuten: Directional Preference und Zentralisationsphänomen.

Joshua Kidd
\end{abstract}

\section{Hintergrund: fehlende Richtlinien für RTP bei LBP}

Bei Assessment und Behandlung von Sportlern mit LBP (Low Back Pain) steht vor allem eine Frage im Vordergrund: Wann kann er oder sie wieder zurück in den Sport? Bis heute gibt es für keine Sportart verbindliche Richtlinien für den RTP, an denen sich der behandelnde Sportphysio orientieren könnte. Das Fehlen von Richtlinien ist wahrscheinlich darauf zurückzuführen, dass zurzeit kaum Fachliteratur zu diesem Thema zur Verfügung steht. Dieser Umstand führt zu Schwierigkeiten bei der Standardisierung $[1,2,3,4,5,6,7,8]$.

Die zweite häufig gestellte Frage lautet: Kann der Sportler trotz Schmerzen spielen? Auf diese Frage gibt es keine einfache Antwort. Zum Beispiel wird Sportlern, bei denen eine Spondylolyse vermutet wird, geraten zu pausieren, wenn Schmerzen auftreten, während Sportler mit chronischem LBP aufgrund einer Muskelzerrung oder Bänderdehnung weiter trainieren und an Wettkämpfen teilnehmen können. Für beide Szenarien gibt es jedoch nur wenig Evidenz [2, 3, 4, 5, 6, 7, 8]. Für Probleme im Bereich der Lendenwirbelsäule wurden auf Expertenmeinungen basierende Richtlinien zum Zeitrahmen für einen RTP veröffentlicht [7]. Diese Richtlinien empfehlen, bei Zerrungen im LWS-Bereich vor dem RTP die Beweglichkeit vollständig wiederherzustellen. Patienten mit Spondylolyse und Spondylolisthese (Stufe 1) sollten vier bis sechs Wochen pausieren und erst wieder spielen, wenn die Beweglichkeit vollständig wiederhergestellt und eine schmerzfreie Extension möglich ist [3]. Sportler mit einem lumbalen Bandscheibenvorfall sollten nach einer Operation sechs bis 12 Wochen pausieren. Nach einer Wirbelsäulenfusion sollte eine Auszeit von einem Jahr bis zur Wiederaufnahme der sportlichen Aktivitäten eingelegt werden [2]. Viele Chirurgen vertreten die Meinung, dass nach einer Wirbelsäulenfusion Kontaktsportarten überhaupt nicht mehr ausgeübt werden sollten [2]. Iwamoto und Kollegen verglichen in einem Review chirurgische und konservative Behandlungsmethoden für lumbale Bandscheibenvorfälle u.a. im Hinblick darauf, wie viel Zeit die Sportler benötigten, um ihr früheres Leistungsniveau wieder zu erreichen [9]. $79 \%$ der konservativ behandelten Sportler benötigten dafür durchschnittlich 4,7 Monate, während 85 \% der Sportler, die durch eine Mikrodisektomie behandelt wurden, 5,2 bis 5,8 Monate benötigten. $69 \%$ der Sportler mit einer perkutanen Disektomie benötigten sieben Wochen bis 12 Monate [9]. Soweit den Auto- ren bekannt ist, basieren diese Richtlinien hauptsächlich auf starren Zeitvorgaben für Heilung und Tests zur Beweglichkeit. Symptomatische, mechanische und funktionelle Befunde wurden dabei nicht berücksichtigt.

Angesichts des Mangels an eindeutiger Evidenz ist es für den behandelnden Physio wichtig, spezifische Kriterien zu finden, welche den zeitlichen Ablauf des RTP definieren. Ein Ansatz, um den richtigen Zeitpunkt für den RTP zu bestimmen, ist die Identifikation von Directional Preference (DP) und Zentralisation. Greg Lynch erörtert im Vertiefungsartikel die Bedeutung von DP. Das Einbeziehen dieser definierten Kriterien könnte ein erster Schritt zur Etablierung funktioneller und verlässlicher Richtlinien für den RTP sein. In diesem Fallbericht stelle ich die Behandlung eines Sportlers mit LBP vor und zeige, wie diese Richtlinien angewandt werden können, um den Sportler wieder auf sein früheres Spielniveau zu bringen.

\section{Der Patient: junger und großer Footballspieler mit BS-Vorfall}

Bei dem Patienten handelte es sich um einen 20-jährigen College-Footballspieler, der vom Mannschaftsorthopäden überwiesen worden war. Diagnostiziert wurden ein „großer“ L5-S1-Bandscheibenvorfall und eine linksseitige S1-Radikulopathie. Der Patient war 1,88 Meter groß und wog 102 Kilogramm. Bei der Erstuntersuchung beim Mannschaftsorthopäden gab er seine Schmerzen mit 8/10 auf einer VAS-Skala an. Die Schmerzen waren in der linken Gesäßhälfte und im linken posterioren Oberschenkel lokalisiert. Sie traten vor zwei Wochen zum ersten Mal auf, nach der Teilnahme an einem Mannschaftstraining, bei dem Gewichte zum Einsatz kamen. Das Programm beinhaltete Kniebeugen mit Gewichten, Gewichtheben (sogenannte Power Cleans), Kreuzheben und plyometrisches Training. Die Schmerzen begannen ungefähr zwei Wochen nach Beginn des Übungsprogramms. Anfänglich verspürte der Sportler einen dumpfen Schmerz im linken unteren Rückenbereich beim Kreuzheben mit 200 Kilogramm. Dieser dumpfe Schmerz nahm immer weiter zu und trat dann auch beim Vorbeugen, beim Laufen und beim plyometrischen Training auf. Der Sportler bemerkte, dass die Schmerzen manchmal beim Stehen, Sitzen und Gehen zunahmen. Außerdem berichtete er, dass die Schmerzen regelmäßig Schlafstörungen zur Folge hatten und frühmorgens schlimmer waren als zu anderen Tageszeiten. Er gab 
ein positives Valsalva-Zeichen an. Außerdem provozierten Husten und Niesen sowie Pressen beim Stuhlgang die Beschwerden. Die größte Schmerzlinderung wurde durch Einnahme der Embryo-Haltung erreicht, vor allem, wenn er auf der linken Seite lag. Weiterhin gab er an, dass er keine Schwäche in den Beinen verspürte, jedoch in den linken hinteren Oberschenkel reichende Parästhesien.

Nach einer Besprechung mit dem Mannschaftsarzt erhielt er eine Packung Prednison - ein Kortisonpräparat - und wurde zur Physiotherapie überwiesen. Seine Vorgeschichte war unauffällig. Zum Zeitpunkt der Erstuntersuchung beim Physiotherapeuten nahm er Prednison und Naproxen sowie ein Muskelrelaxans. Er hatte sich nie einer Operation unterziehen müssen, keine schwerwiegenden Stürze oder Unfälle erlitten und gab keinen bösartigen Nachtschmerz an. Wegen eines Problems mit der rechten Schulter, das durch konservative Behandlung gelöst werden konnte, war er vor zwei Jahren physiotherapeutisch behandelt worden. Abgesehen von den Medikamenten war sein aktueller LBP bis zu diesem Zeitpunkt nicht behandelt worden.

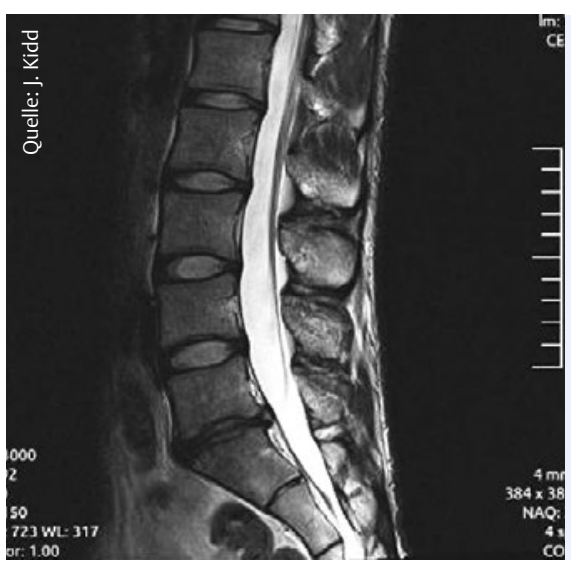

Abb. 1 Das MRT des 20-jährigen Footballspielers zeigt eine große posteriore Bandscheibenextrusion.

\section{Klinische Untersuchung}

\section{Erstuntersuchung}

Die physiotherapeutische Erstuntersuchung wurde von einem Fellow of the American Academy of Orthopedic and Manual Physical Therapy mit MDT-Diplom durchgeführt. Wie bereits beschrieben, wurde dokumentiert, dass die Schmerzen im Rücken und im linken Bein auftraten. Der Roland-Morris-Funktionsfragebogen ergab 16/24. Das MRT zeigte eine große posteriore Bandscheibenextrusion, die praktisch den gesamten Spinalkanal auf der linken Seite ausfüllte (Abb. 1).

Bei der Untersuchung des Patienten ging der Therapeut nach dem MDT-Untersuchungssystem vor (wie von Robin McKenzie beschrieben [10]). Die Körperhaltung des Patienten war leicht gekrümmt und durch eine deutlich reduzierte Lumballordose gekennzeichnet. Es waren keine akuten Deformierungen wie „akute Kyphose“ oder „akuter lateraler Shift“ zu erkennen. Eine erste Korrektur der Körperhaltung hatte keine Wirkung auf die Symptome.

Die neurologische Untersuchung zeigte einen bei $30^{\circ}$ positiven SLR (Straight Leg Raise), der die Symptome im linken Bein hervorrief. Der Achillessehnenreflex links war abgeschwächt. Alle anderen Reflexe waren unauffällig. Außerdem wurde eine umlaufende Taubheit in der linken Wade und dem linken Fuß festgestellt. Manuelle Muskeltests ergaben 5/5 in beiden Beinen für alle Hauptmuskelgruppen. Eine einbeinige Kniebeuge war jedoch links nicht möglich.

Die Beweglichkeit der Wirbelsäule im Stehen wurde gemessen und entsprechend dem MDT-Untersuchungsformular qualitativ bewertet (d.h. minimale, moderate oder schwere Restriktion in den sagittalen und frontalen Ebenen). Bei allen Bewegungen in der sagittalen und frontalen Ebene war die Beweglichkeit reduziert. Ebenso stellte der Physio starke Einschränkungen bei Extension (Abb. 2) und Seitgleiten nach links (Abb. 3) fest. Flexion (Abb. 4) und Seitgleiten nach rechts (Abb. 5) wiesen kleinere Einschränkungen auf. Flexion, Extension und Seitgleiten nach rechts
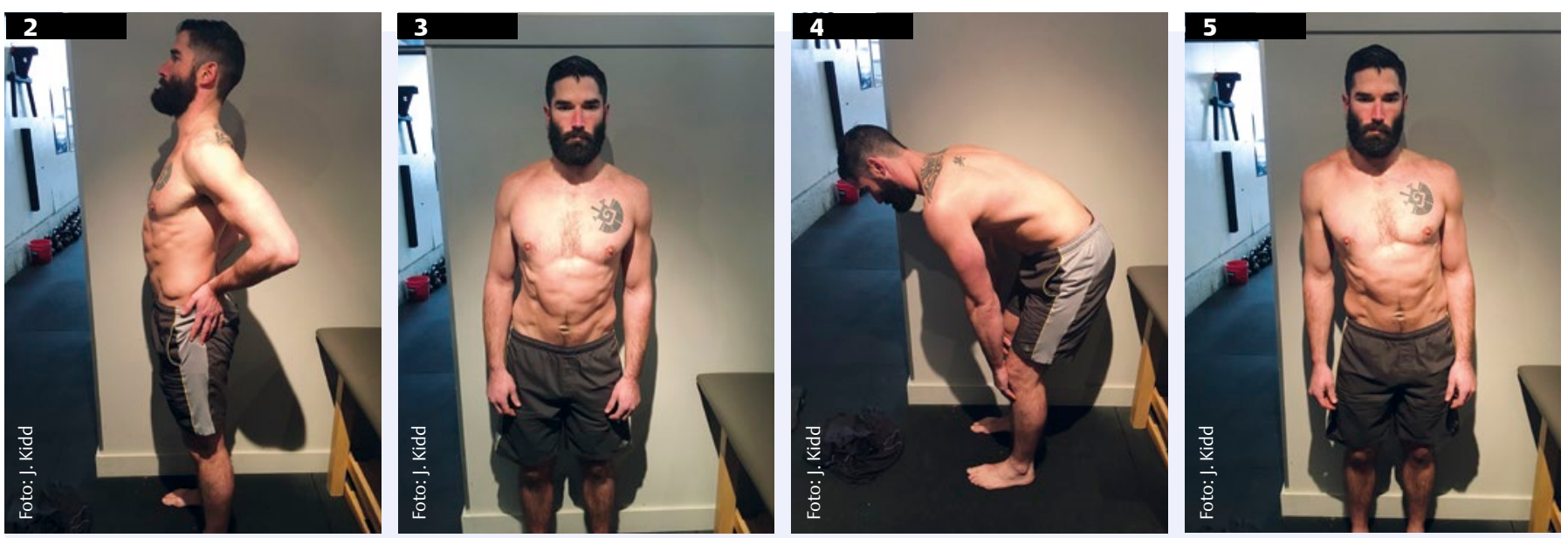

Abb. 2 Bei der Untersuchung der WS-Beweglichkeit in Extension fanden sich starke Einschränkungen, ...

Abb. 3 ... ebenso beim Seitgleiten nach links.

Abb. 4 Flexion ...

Abb. 5 ... und Seitgleiten nach rechts zeigten kleinere Einschränkungen. 


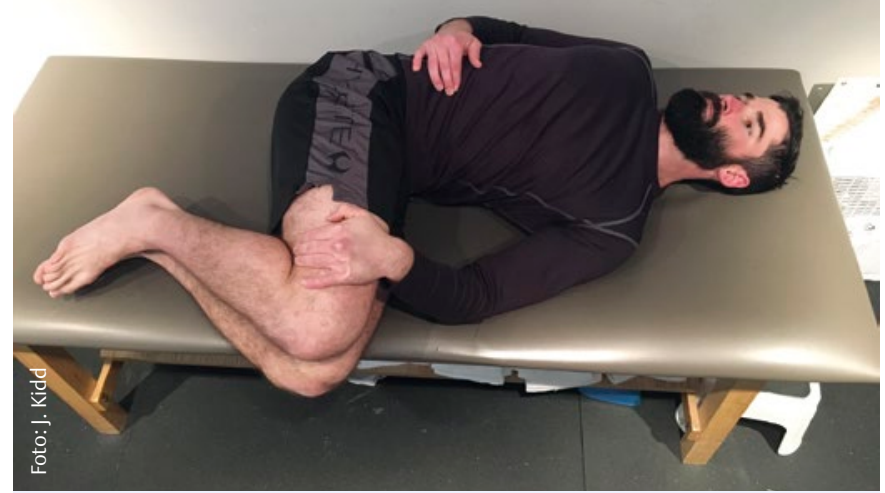

Abb. 6 Directional Preference: gehaltene lumbale Rotation in Flexion

führten alle zu einer Zunahme der bekannten Schmerzen im Bein. Seitgleiten nach links (der schmerzhaften Seite des Patienten) reduzierte die Schmerzen kurzfristig. Als er in die Ausgangsposition zurückkehrte, war jedoch keine Verbesserung der Symptome zu beobachten.

Einer der wichtigsten Aspekte eines MDT-Assessments ist die Reaktion des Patienten auf wiederholte Bewegungen, durch die eine Zentralisation der Schmerzen oder eine Directional Preference (DP) identifiziert werden soll. Auf Grundlage der bisherigen Erkenntnisse zum Patienten und den Prinzipien der MDT instruierte der Therapeut den Patienten, mehrere Extensionen im Liegen hintereinander auszuführen. Nach zehn Wiederholungen berichtete der Patient eine Schmerzzunahme im linken Bein, was als Verschlimmerung der Symptome interpretiert wurde. Angesichts dieser offensichtlichen Verschlimmerung der Symptome bei Extensionsbewegungen beschloss der Therapeut, den Einfluss wiederholter Bewegungen in der frontalen Ebene (Seitgleiten im Stehen) zu untersuchen. Diese klinische Entscheidung basierte auf der Reaktion des Patienten auf Extensionsbewegungen und auf seiner früheren Reaktion auf Belastungsstrategien in der frontalen Ebene (Schmerz eliminiert, jedoch nicht bleibend besser). Eine einmalige Wiederholung des Seitgleitens im Stehen (SGIS) nach links rief dieselbe Reaktion wie bei der Testung der Beweglichkeit hervor (d.h. Eliminierung der Schmerzen), aber wieder war nach der einmaligen Wiederholung keine Verbesserung zu beobachten. Auf Grundlage dieser Reaktion wurde der Patient instruiert, mehrere SGIS nach links auszuführen. Nach 15 Wiederholungen kehrten die Schmerzen in der linken Gesäßhälfte immer wieder zurück, nachdem die Bewegung beendet worden war. Diese Strategie brachte also keine bleibende Verbesserung. Um Bewegungen in der frontalen Ebene weiter zu untersuchen, wurde der Patient gebeten, zwei Minuten lang eine gehaltene Rotation in Flexion mit den Knien nach links auszuführen (Abb. 6). Der Patient berichtete, dass während dieser Zeit Schmerzen in der linken Gesäßhälfte verschwanden und auch nicht wiederkehrten, nachdem er in die Ausgangsposition zurückgekehrt war.

\section{Klinische Schlussfolgerung}

Die Reaktion des Patienten auf wiederholte Bewegungen in der sagittalen und frontalen Ebene zeigen, dass bei ihm eine Zentralisation durch Bewegungen in der frontalen Ebene vorliegt. Darüber hinaus hatten die Belastungsstrategien in der sagittalen Ebene unerwünschte Reaktionen hervorgerufen. Positive, wenn auch nicht nachhaltige Veränderungen waren in der frontalen Ebene aufgetreten, wenn der Patient eine gewichttragende Haltung (Stehen) einnahm.

Die vorläufige Klassifikation dieses Patienten im MDT-System ist: Derangement, unilaterale Schmerzen oberhalb des Knies.

\section{Erste Intervention}

Auf Grundlage der Reaktion während der Tests mit wiederholten Bewegungen entschied sich der Therapeut für eine lumbale Rotation in Flexion mit den Knien nach links (Abb. 6). Er wählte eine unbelastete Ausgangsstellung, weil - wie bereits beschrieben - Seitgleiten im Stehen eine Zunahme der Symptome verursachte und die unbelastete Bewegung, Rotation nach links, zur erwünschten Reaktion führte. Diese kombinierte Bewegung eliminierte die Schmerzen des Patienten vollständig und bewirkte eine signifikante Verbesserung der Beweglichkeit in der sagittalen Ebene, als der Patient erneut im Stehen getestet wurde. Der Patient erhielt Instruktionen zur Verbesserung seiner Körperhaltung und ein Übungsprogramm, das nur eine einzige Übung umfasste (gehaltene Rotation für 2 Minuten mit den Knien nach links), die sechs bis acht Mal pro Tag ausgeführt werden sollte. Anschließend wurde der Patient nach Hause geschickt.

\section{Behandlungsverlauf}

\section{Behandlungstermin}

Der Patient kehrte zwei Tage später zurück und berichtete, er sei fast schmerzfrei und verspüre lediglich morgens eine leichte „Steifheit“. Die Schmerzbehandlung durch die Rotation-in-Flexion-Übung hatte gut angeschlagen. Die Messung der Beweglichkeit lieferte nun sehr gute Ergebnisse, auch wenn Flexion, Extension und Seitgleiten nach links immer noch leicht eingeschränkt waren. Angesichts dieser Fortschritte ordnete der Therapeut Bewegungen in der sagittalen Ebene gemäß den MDT-Behandlungsprinzipien an. Nach der guten Reaktion des Patienten auf unbelastete Übungen wurden auch Bewegungen in der sagittalen Ebene ohne Gewichtsbelastung in Angriff genommen. Der Patient führte wiederholte Extensionen im Liegen durch. Nach vier Sätzen mit je zehn Wiederholungen gab der Patient an, dass die Schmerzen verschwunden wären. Die anschließende Untersuchung ergab, dass die Beweglichkeit in allen Ebenen fast vollständig wiederhergestellt war, mit Ausnahme der Flexion, die immer noch Schmerzen im linken Gesäßbereich verursachte. Angesichts seiner positiven Reaktion auf die Extensionsübungen im Liegen wurde der Patient instruiert, diese weiter durchzuführen und nach wie vor an seiner Körperhaltung zu arbeiten.

\section{Behandlungstermin}

Nach drei Tagen erschien der Patient wieder und berichtete, dass die positive Entwicklung seiner Symptome weiter voranging, er 


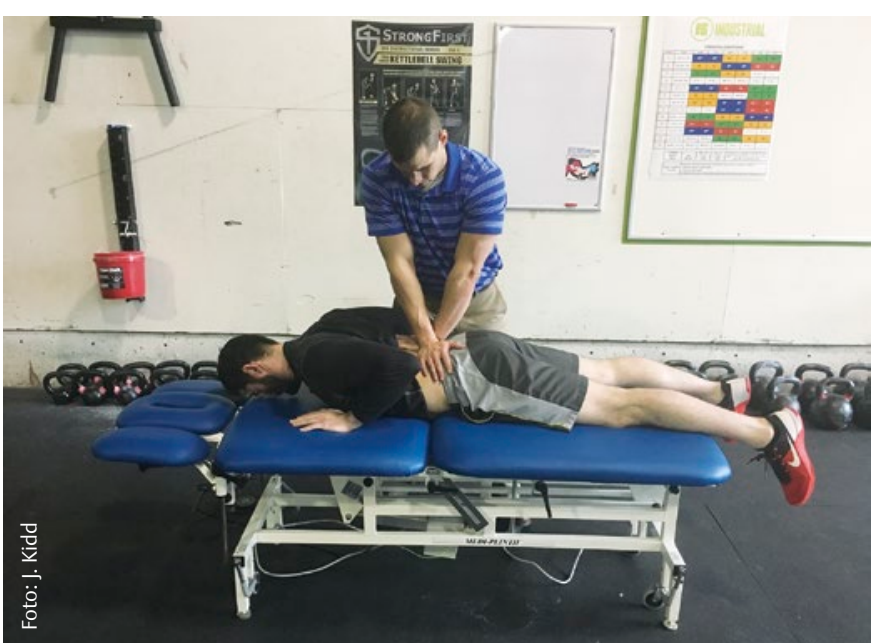

Abb. 7 Extension mit Überdruck durch den Therapeuten

aber immer noch an zeitweilig auftretender Steifheit in der linken unteren LWS-Bereich litt, vor allem während der Morgenstunden. Abgesehen von einer leichten Einschränkung der Extension war die Beweglichkeit nun in allen Bewegungsebenen normal. Die Übung, die er zu Hause praktizierte, hatte mittlerweile keine Wirkung mehr auf die Symptome. Angesichts der Stagnation der Symptome entschied sich der Therapeut, den Patienten die Übung mit manuellem Überdruck durch den Therapeuten ausführen zu lassen. Nach 20 Wiederholungen waren die Schmerzen vollständig eliminiert, und die Beweglichkeit war in sämtlichen Ebenen wiederhergestellt. Der Therapeut zeigte ihm, wie er diesen Überdruck selbst erzeugen könne, und wies ihn an, diese Übung weiterhin zu Hause zu praktizieren. 24 Stunden später fand die nächste Untersuchung statt, die zeigte, dass seine lumbale Mobilität nun wieder völlig schmerzfrei war.

\section{Return to Sport}

Die MDT-Prinzipien für die Behandlung eines Derangements umfassen vier Behandlungsphasen: (1) Reduktion, (2) Erhaltung der Reduktion, (3) Funktionswiederherstellung und (4) Prophylaxe. Angesichts des aktuellen Status des Patienten und der dauerhaften symptomatischen und mechanischen Verbesserungen (Beweglichkeit) wurde nun die dritte Phase - Funktionswiederherstellung - eingeleitet. Im Allgemeinen versteht man unter „Funktionswiederherstellung“ die Wiederherstellung aller Bewegungen, die zuvor eingeschränkt waren und Schmerzen verursachten, gefolgt von der Rückkehr zu normaler Aktivität. Bei Sportlern müssen außerdem Aspekte berücksichtigt werden wie die spezifischen Anforderungen ihrer Sportart, das Trainingsprogramm in der spielfreien Zeit und die Einstellung und Überzeugungen des Sportlers in Bezug auf seine Verletzung. Zunächst wurde seine Bewegungsfähigkeit in allen Ebenen untersucht und als vollständig und schmerzfrei eingestuft (Abb. 8-11). Der SLR war nun bei $90^{\circ}$, und der Patient war in der Lage, Beugebewegungen mehrfach auszuführen, ohne dass Symptome auftraten. Danach führte der Therapeut mit ihm ein ausführliches Gespräch über seine Einstellung gegenüber seiner Verletzung. Dieses Gespräch diente dazu, kein angstbedingtes Vermeidungsverhalten seitens des Sportlers aufkommen zu lassen und sein Selbstbewusstsein im Hinblick auf seinen RTS zu stärken. Gegenstand dieses Gesprächs war auch die Fachliteratur, der zu entnehmen ist, dass abnormale MRT-Befunde bei asymptomatischen Personen nicht ungewöhnlich sind [11]. Zuletzt wurde das Trainingsprogramm des Patienten evaluiert. Wichtige Aspekte waren dabei die verschiedenen Trainingsaktivitäten, der Trainingsumfang sowie Häufigkeit, Intensität und Zeitplan des Trainings. Zur Sprache kamen auch die außersportlichen Aktivitäten des Patienten wie berufliche Anforderungen und Freizeitbeschäftigungen. Auf Grundlage dieser Informationen und in Absprache mit seinem Kraft- und Ausdauertrainer wurde ein abgestuftes Belastungsprogramm für den Sportler entwickelt.
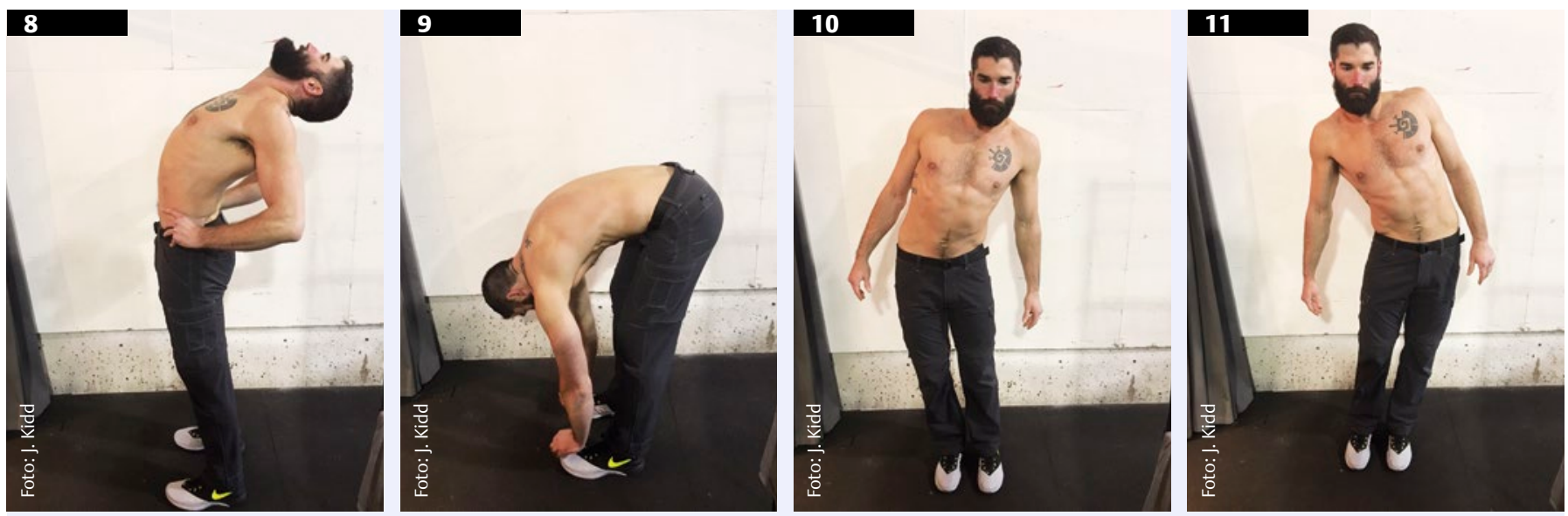

Abb. 8-11 Nach der 2. Sitzung wurde die Bewegungsfähigkeit nochmal in allen Ebenen untersucht und als vollständig und schmerzfrei eingestuft. 

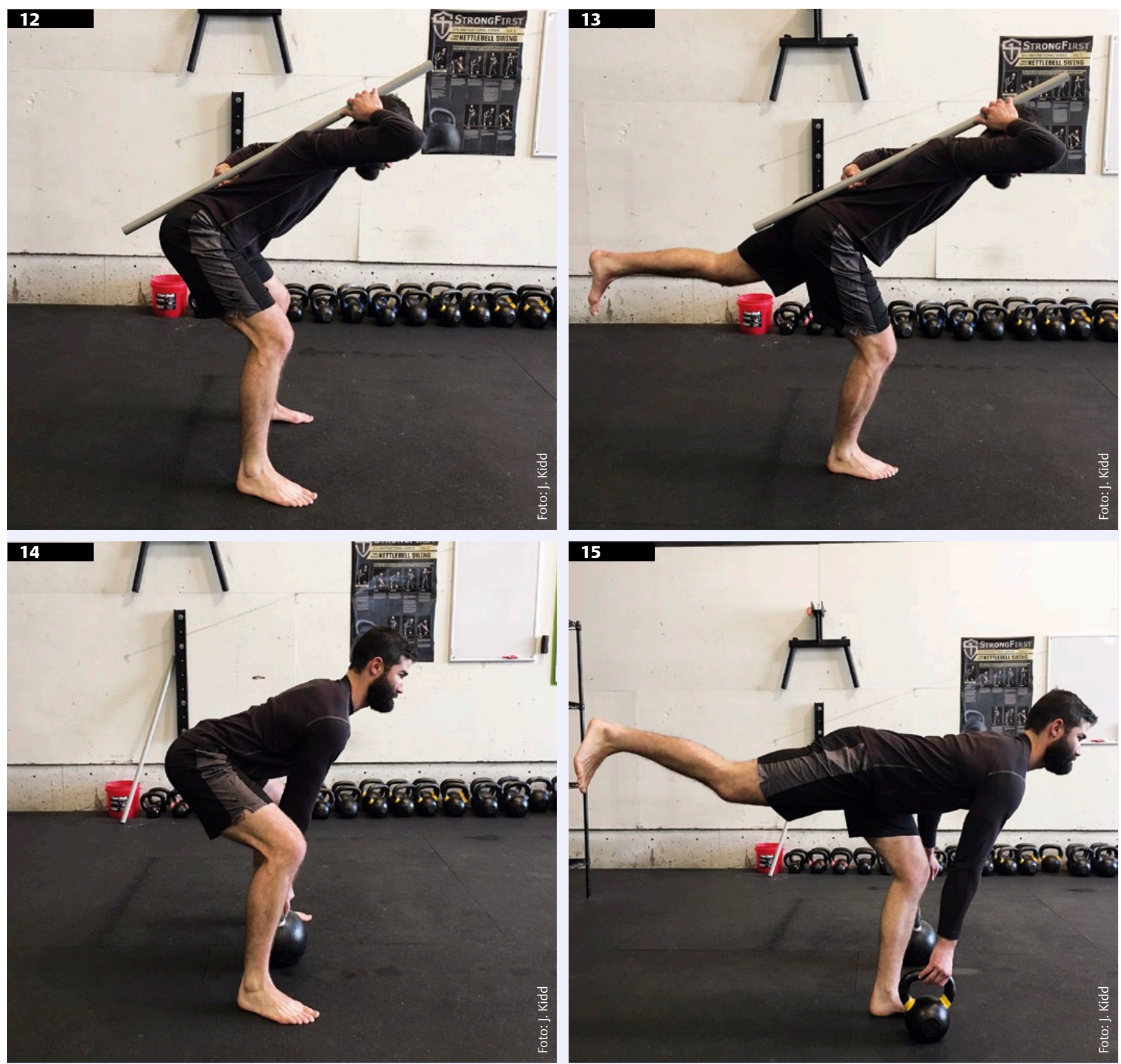

15

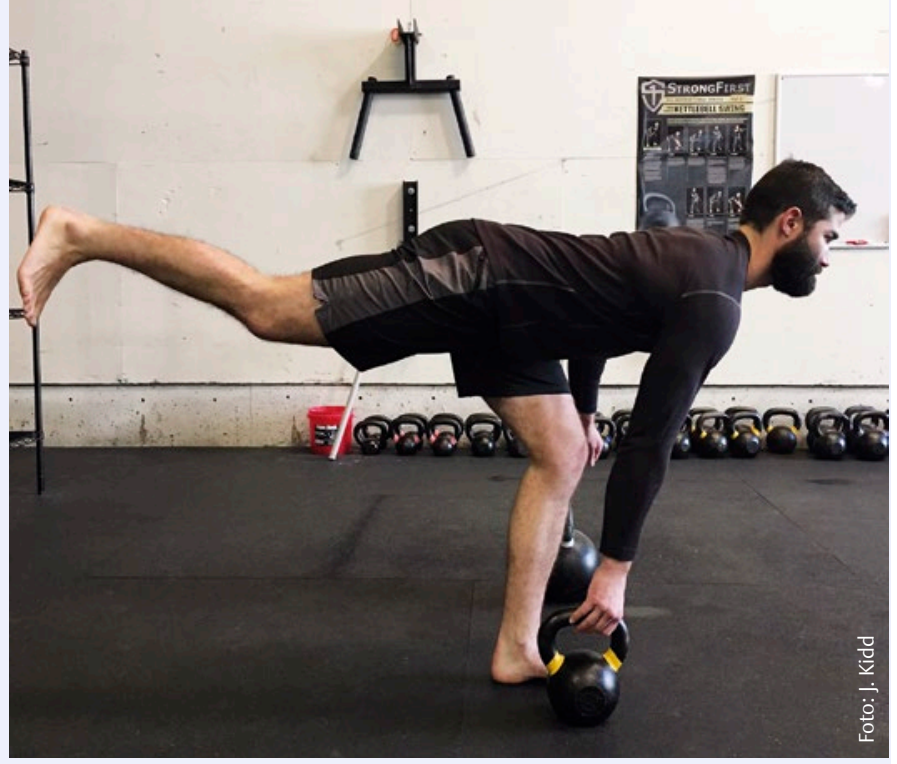

Abb. 12 6. Behandlungstermin: Hüftflexion (Hip Hinge) mit Stab zur Kontrolle der WS-Haltung

Abb. 13 7. Behandlungstermin: Hüftflexion auf einem Bein (Single Leg Hip Hinge)

Die Auswahlkriterien und die Durchführungsweise der Übungen und Belastungsstrategien folgen, wie bereits erwähnt, den MDT-Prinzipien für die Behandlung eines Derangements (3. Behandlungsphase). Aufgrund der limitierten Evidenz zum Thema RTP und zur Ausführung von Bewegungen mit hohem Kraftaufwand wurden die symptomatischen (Lokalisierung der Symptome) und mechanischen (Kraft, Beweglichkeit, neurale Spannung)

Wiederbefunde des Patienten sowohl vor als auch nach Ausführung der Übungsbewegungen evaluiert. Wenn der Patient in der Lage war, die Bewegungen auszuführen, ohne dass sich die Wiederbefundwerte verschlechterten, wurde der Schwierigkeitsgrad der Übungen erhöht: von Bewegungen in einer Ebene zu Bewegungen in mehreren Ebenen, von Übungen auf beiden Beinen zu Übungen auf einem Bein, von unbelasteten zu belasteten Bewe- hantel (Kettlebell Deadlift) und Kreuzheben mit Kugelhantel auf einem Bein (Kettlebell Single Leg Deadlift) 


\section{LINKS}

Video 1 zum Kettlebell Swings aus dem 9. Behandlungstermin

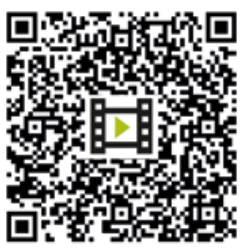

LINKS

Video 3: Kreuzheben mit Langhantel (Barbell Deadlift)

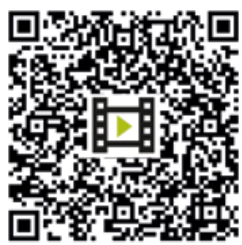

\section{LINKS}

Video 5: Jerks

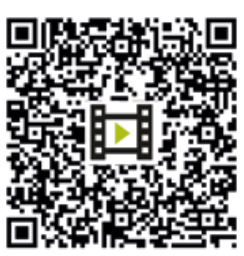

gungen, von langsam/kontrolliert zu plyometrisch. Im Einzelnen handelte es sich bei den Übungen um:

4.und 5. Behandlungstermin: wiederholte Flexion im Liegen, Steigerung zu wiederholter Flexion im Stand

\section{6. -8. Behandlungstermin (s. Fotos Seite 76)}

9. Behandlungstermin

Kugelhantel-Schwingen (Kettlebell Swings) (s. Video 1)

„Turkish Get Up” (s. Video 2)

10. Behandlungstermin

Kreuzheben mit Langhantel (Barbell Deadlift) (s. Video 3)

Kugelhantel-Reißen (Kettlebell Snatches) (s. Video 4)

11. Behandlungstermin

Jerks (s. Video 5)

12. Behandlungstermin

Schlittenschieben (Sled Push) (s. Video 6)

Während der gesamten Rehabilitationsdauer blieb der Therapeut in Kontakt mit dem Kraft- und Ausdauertrainer des Sportlers, um mögliche Probleme bereits im Vorfeld auszuräumen bzw. gemeinsam zu lösen.

\section{Abschluss der Therapie}

Zum Zeitpunkt der letzten Sitzung, vier Wochen nach Beginn der Physiotherapie, konnte der Sportler wieder an allen Trainingsaktivitäten der spielfreien Zeit teilnehmen. Beim Roland-MorrisFunktionsfragebogen (RMQ) schnitt er mit 0/24 ab. Die minimal bedeutsame klinische Differenz für den RMQ liegt nachweislich

\section{LINKS}

Video 2 zum Turkish Get Up: eine Kettlebell-Übung aus dem 9. Behandlungstermin

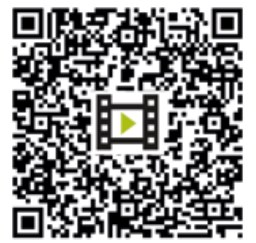

\section{LINKS}

Video 4: Kugelhantel-Reißen (Kettlebell Snatches)

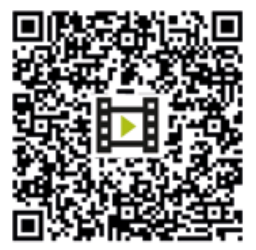

\section{LINKS}

Video 6: Schlittenschieben (Sled Push)

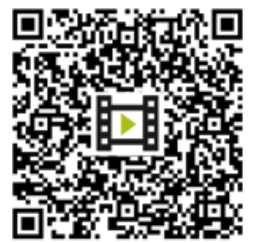

bei 5 Punkten für die gesamte Skala [12]. Nach seiner Entlassung aus der Physiotherapie nahm der Sportler noch zwei weitere Male Kontakt zum Therapeuten auf: nach Beginn des Trainings für die kommenden Spiele und während seiner Teilnahme am ersten Spiel. Beim ersten Besuch berichtete er, dass er keine Schmerzen mehr verspüre, aber ein gelegentliches leichtes „Kribbeln“ im linken Bein, das nach seinen Workouts auftrat. Bei seinem letzten Besuch war er, abgesehen von leichtem LBP nach einem Spiel, den er mithilfe seiner Übungen problemlos behandeln konnte, symptomfrei.

\section{Diskussion}

Aufgrund des Mangels an Evidenz ist es schwierig, standardisierte Richtlinien für den RTP bei LBP aufzustellen. Während man für die Entwicklung von RTP-Richtlinien bei Verletzungen der unteren Extremität auf zahlreiche Fachartikel zurückgreifen kann (FMS, Hüpftest, Star Excursion Balance Test, Y-Balance-Test), ist es bisher nicht gelungen, verbindliche Richtlinien für LBP aufzustellen [13]. Zwar stehen viele funktionelle Leistungstests zur Verfügung, die dazu beitragen können, den richtigen Zeitpunkt für die Rückkehr zu uneingeschränkter Aktivität zu ermitteln, aber es ist nur wenig darüber bekannt, in welchem Verhältnis die verschiedenen Tests zueinander stehen und welche Tests besser einzeln oder in Kombination mit anderen Tests angewandt werden sollten, um ein möglichst breites Spektrum an Funktionen zu untersuchen [13]. Aufgrund der fehlenden Standardisierung 
des RTP ist es wichtig, die symptomatischen, mechanischen und funktionellen Wiederbefunde zu überwachen, sodass sie als Orientierungspunkte für den Rehabilitationsprozess dienen können. Ein neuartiger Ansatz für den RTP könnte die Einbeziehung von Directional Preference und Zentralisation sein. Findet man diese Phänomene, so sind sie aussagekräftige Prädiktoren für Schmerzen und funktionelle Ergebnisse $[14,15,16]$. Treten sie nicht auf, ist die Prognose eher unsicher $[14,15,16]$. Aufgrund der Menge an Fachliteratur, die diese starken Prädiktoren stützt, ist es wichtig, ihren potenziellen Nutzen für die Arbeit mit Sportlern anzuerkennen. Wie in diesem Fallbericht gezeigt wurde, kamen immer dann, wenn im Verlauf der Rehabilitation eine Verschlechterung der Wiederbefunde eintrat, die symptomreduzierenden Übungen zum Einsatz, und das Trainingsregime wurde modifiziert, um eine kontinuierliche Verschlechterung zu verhindern. Sobald der Sportler in der Lage war, alle sportspezifischen Aktivitäten ohne Verschlechterung der oben genannten Wiederbefunde auszuführen, konnte er ohne Beschwerden seine früheren Aktivitäten wieder aufnehmen.

\section{:: Ein neuartiger Ansatz für den RTP könnte die}

Einbeziehung von Directional Preference und

\section{Zentralisation sein.}

Könnten diese Prinzipien auch auf den RTP angewendet werden? Vielleicht ist ein Paradigmenwechsel in unserem Denken nötig, in der Form, dass wir uns stärker an den symptomatischen und mechanischen Wiederbefunden orientieren als an pathoanatomischen Befunden und an auf starren Zeitvorgaben basierenden Protokollen. Die Identifikation von Directional Preference und Zentralisation könnte eine angemessenere Vorgehensweise für die Behandlung von Sportlern sein, sowohl für das Training während der Saison als auch für das Training in der spielfreien Zeit. Dieser Fallbericht zeigt, wie eine auf den Reaktionen eines Sportlers basierende Methode, bei der Directional Preference und Zentralisation im Mittelpunkt stehen, für die Sportlerpopulation insgesamt von Nutzen sein könnte.

\section{Take Home Message}

- „Wiederholte Bewegungstests“ können sowohl in der Diagnostik als auch in der Therapie von Sportlern sehr nützlich sein.

- Directional Preference und Zentralisation bieten einen neuen Ansatz, um den Zeitpunkt für Return to Sport von Rückenpatienten zu bestimmen.

- Mit dem Wissen um die passende Behandlungsstrategie können sich Sportler auch während eines Wettkampfs effektiv selbst behandeln.

\section{Literatur}

1 Petering $R$, Webb C. Treatment options for low back pain in athletes. Sports Health 2011; 3: 550-555

2 Bono C. Low back pain in athletes. J Bone Joint Surg Am 2004; 86: 382-396

3 d'Hemecourt PA, Gerbino II PG, Micheli LJ. Back injuries in the young athlete. Clin Sports Med 2000; 19: 663-679

4 Granhed H, Morelli B. Low back pain among retired wrestlers and heavyweight lifters. Am J Sports Med 1988; 16: 530-533

5 Sward L, Hellstrom M, Jacobsson B et al. Disc degeneration and associated abnormalities of the spine in elite gymnasts: A magnetic resonance imaging study. Spine 1991; 16: 437-443

6 Kujala UM, Taimela S, Erkintalo M et al. Low-back pain in adolescent athletes. Med Sci Sports Exerc 1996; 28: 165-170

7 Eck JC, Riley LH III. Return to play after lumbar spine conditions and surgeries. Clin Sports Med 2004; 23: 367-379

8 French SD, Cameron M, Walker BF et al. A Cochrane review of superficial heat or cold for low back pain. Spine 2006; 31: 998-1006

9 Iwamoto J, Sato Y, Takeda T et al. The return to sports activity after conservative or surgical treatment in athletes with lumbar disk herniation. Am J Phys Med Rehabil 2010; 89: 1030-1035

10 McKenzie R, May S. The lumbar spine: Mechanical diagnosis and therapy. 2nd ed. Waikanae 2003. New Zealand: Spinal Publications

11 Alyas F et al. MRI findings in the lumbar spines of asymptomatic, adolescent, elite tennis players. Brit J Sports Med. 2013; 4: 836-841

12 Stratford PW, Binkley JM, Riddle D et al. Sensitivity to change of the Roland-Morris back pain questionnaire: Part 1. Phys Ther 1998; 78: 11861196

13 Manske R. Functional performance testing for power and return to sports. Sports Health 2013; 5: 244-250

14 George SZ, Bialosky JE, Donald DA. The centralization phenomenon and fear-avoidance beliefs as prognostic factors for acute low back pain: A preliminary investigation involving patients classified for specific exercise. $J$ Orthop Sports Phys Ther 2005; 35: 580-588

15 Long A, May S, Fung T. The comparative prognostic value of directional preference and centralization: A useful tool for front-line clinicians? J Manual Manip Thera 2008; 16: 248-254

16 May S, Aina A. Centralization and directional preference: A systematic review. Manual Therapy 2012; 17: 497-506

\section{AUTOR}

Joshua Kidd, PT, DPT, OCS, Dip. MDT, MOMT, CSCS, FAAOMPT promovierte 2007 in Physical Therapy. Seit 2010 ist er als orthopädischer Spezialist zugelassen. 2011 absolvierte er die Diploma-Ausbildung in MDT, erlangte 2015 den Master in Orthopedic Manual Therapy und beendete 2016 sein Fellowship Training.

Josh betreibt klinische Forschung und hat im „Journal of Manual and Manipulative Therapy“ publiziert.

Außerdem ist er Clinical Mentor im „MDT Orthopedic Residency and Fellowship“-Programm.

Im Zentrum „Advance Sports and Spine Physical Therapy“ in Portland, Oregon behandelt Josh College- und Profisportler.

E-mail:josh@advancesportsandspine.com

\section{BIBLIOGRAFIE}

DOI 10.1055/s-0043-105602

Sportphysio 2017; 5: 72-78

(C) Georg Thieme Verlag KG

Stuttgart · New York · ISSN 2196-5951 